\title{
Study of Cellular Immune Function and Regulation Measurement
}

\author{
Qingfeng Wan ${ }^{1}$, Wenbin Zhang ${ }^{2}$ \\ ${ }^{1}$ Jiangxi Medical College, Shangrao, Jiangxi, 334000 \\ ${ }^{2}$ Guangfeng People's Hospital, Shangrao, Jiangxi, 334600 \\ a email
}

KEYWORDS: Cell; Immunity; Adhesion; Regulation

\begin{abstract}
Cellular immune system" concept first proposed in 1981 by the American scholar Siegel. 20 years of domestic and foreign scholars have done a lot of research and found that there are many cells and immune-related substances, and have recognition, adhesion, concentrated, anti-antigen, the antigen transfer information and scavenging of circulating immune complexes. Not only can the immune cells involved in the regulation, and a complete system of self-regulation in the pathogenesis of many diseases, the immune-deficient cells plays an important role. The article reviews the development process of immune cell research, described the material basis of cellular immunity, immune function and factors affecting cellular immune function, the researchers reviewed the progress in all aspects of cellular immunity molecular biology, clinical medicine, Chinese medicine and the like.
\end{abstract}

\section{Introduction}

In the 1930s, it found that different people have different cell adhesion function. 1950s, found phagocytic cells promote the role, having adhered immune complexes (IC) functions are part of the body's defense mechanism. 1960s, and it confirms this human immune cells have adhesion function is achieved through C3 receptors. 1970s, for the first time in human cell membranes isolated and purified complement receptor type I, and a detailed study of the characteristics of this receptor found on the cell membrane, although much lower than the density of CR1 white blood cells, but the total number of circulating white blood cells are much more than about 1000 times, so more than $85 \%$ of CR1 are in the cell membrane. 80s, at the conclusion based on the results of previous studies, Siegel presented the new concept of "cellular immune system", has opened up new areas of immunology research. Domestic and foreign scholars on cellular immunity deepening found cells CR1, CD58, CD59, DAF, HRF, PIF, NKEF, SOD and peroxidase enzymes and a variety of immune-related substances. There B- endorphin receptor cells, B- adrenergic receptors. They are under the control of neuroendocrine, extensive involvement of non-specific and specific immune response; anti-infective, anti-tumor immune response and homeostasis and regulation has an important role. China since 1982 to study cellular immunity, made a lot of breakthroughs in basic theory and applied research in cellular immunity.

In theory, there is a discovery of serum $58{ }^{\circ} \mathrm{C}, 30$ min does not inactivate cellular immune adhesion promoting factor; cells of various tumor cell immune adhering closely related to cellular immunity and cell adhesion ability of tumor cells CR1 activity; as well as the phenomenon of innate immune cells and so on. In the application, we proposed different methods of measuring cellular immune function improved, and the establishment of a number of monitoring methods of cellular 
immune function. Liu Jingtian confirmed the presence of both positive and negative serum cell regulatory factor, granulocyte cells, lymphocytes (mainly B lymphocytes) have the same regulatory role. We hypothesize that the factor of having a CR1 cells have a regulatory role, it will be such a factor called CR1 immune regulator. Which has a positive regulatory role are known as CR1 immune adherence-promoting factor, a negative regulatory role are called CR1 immune adherence inhibitor. In recent years, researches on cellular immunity have been down to the molecular level, and there are always new discoveries.

\section{Factors Affecting Cell Immunity}

At present, domestic and foreign scholars on many factors affect the cellular immune function (EIF) conducted a more extensive study, mainly as follows:

CR1 has three genotypes: expression type (HH), the expression of type (HL), low expression type (LL). Some scholars have suggested that the normal cell CR1 is subject to genetic control, but not in patients with the genetic control of cell CR1 levels are available; another blood type is heritable TRADITIONAL AND ABO blood group and other observations of the normal crowd of 140 cases EIF, CR1 expression of the activity of different blood there are some differences to B and $\mathrm{AB}$ for the weak.

Cellular immune promoting factor and inhibiting factors are positive and negative regulatory role plays, significantly greater than the effect of promoting factors inhibiting factor; and promoting factor receptor inhibition of disease, inhibition of factor activity increased, along with disease outcome was a certain degree of volatility.

Mainly gender and age, the same age group was slightly lower than male female EIF, but no significant difference. 18 to 40 years of age EIF strongest, with age, the body function decreased, CR1 activity and number were damaged. Into old age, especially after 70 years, EIF decreased.

Lipid peroxide (LPO) can cause many types of cell damage and destruction of cell membrane of the strongest. Acute liver injury and spleen, serum and spleen tissues LPO increased significantly, the cell surface CR1 significantly reduced, while increasing the body's CIC, EIF reduced; superoxide dismutase (SOD) can scavenge oxygen free produced during inflammatory reactions group, in favor of cell surface SOD activity play, diseases (such as epidemic hemorrhagic fever), the serum SOD levels were significantly decreased, due to the deposition of CIC, the cell surface damage SOD activity, resulting in an increase in blood concentrations of free radicals and LPO , damage cell membranes, etc., causing tissue damage, increased reduce the EIF.

By observing different disease process, scholars have come to a common conclusion: EIF with disease outcome and was fluctuated, with the ease of the disease and there is some improvement, with the recurrence of the disease, the deterioration and decline, with the disease recovery and normal or nearly normal.

TDP radiation can increase the number of blood cells and enhances the activity of CR1, excessive UV exposure can damage the EIF, and the right amount of ultraviolet light (in 0.25mED optimal) radiation can be improved EIF. High blood oxygen therapy can significantly improve the CRI of activity in the short term, oxygenated autologous blood transfusion may also improve EIF. Chinese medicine research become a feature of our study of the EIF. Cell membrane glycoprotein receptors, and traditional Chinese medicine to enhance the immune modulators are glycoproteins substances. Severe burn patients, allicin and so bear fruit treat cancer patients in clinical treatment with astragalus. TCS, Angelica, Ginkgo biloba, astragalus polysaccharide, lentinan, curcumin, wolfberry can effectively enhance immune cell function; others, such as acupuncture can improve the body EIF, in complex slide acupuncture points and Shenshu best. 
Since CR1 3 different genetic expression, reflected in its activity is different in different regions. Hypoxia at high altitude, high-altitude radiation damages living in different age groups and immune function in patients with high altitude, EIF has also been varying degrees of damage. Psychological factors, such as the long period of tension, fatigue and other stress, can regulate the immune status of the network through the neuroendocrine system, thus indirectly affecting the body EIF. In the cold stimulation, the body's immune function before showing a transient enhanced immune function, and then quickly turned to plummet. At high temperatures, as the prolonged heat exposure, the immune cell adhesion enhancement is the body a compensatory phenomenon to heat stress response, has a positive effect on the body.

In summary, many factors affect the EIF, according to EIF can determine the status of the immune system for clinical diagnosis and treatment guidelines, and to explore the status and role of the causal mechanisms of cellular immune regulation in the field is important.

\section{Study on Cellular Immunity in Various Fields}

Molecular biology of cellular immunity that people focused on the following areas: cell damage can produce tumor necrosis factor-inducible factor (TNFIF), is a self monocytes can stimulate the release of a large number of specific factors. This new synthesis may be the result of biochemical modifications occur in the stimulation of cell membrane composition and other physical and chemical factors, and guess GPI on the cell membrane may be related, but their molecular structure and distribution have not yet been defined. Normal cells contain a large number of NK cell cytoplasm enhancement factor (NKEF) and the expression of cell survival with prolonged low molecular weight and increase phagocytosis inhibitory factor (PIF), and other cytokines. RBC cytokine involved in antiviral activity of interferon (IFN), the activity of a cytokine-mediated inflammation TNF, IL-6, IL-8, active cells of hematopoietic growth factor CSF, IL-3 and other cytokines in the regulation.

Since the 1982 invention of the cell C3b receptors and immune complex rosette test, people have established a cell SPA rosette test, monoclonal antibodies Coomb's test, adjustment factor activity was measured, zymosan hemagglutination method, tumor hemagglutination method more than 10 kinds of methods. In recent years, at home and abroad and the establishment of a cell membrane and promote phagocytosis chemiluminescence method, determination of cell C3b receptor in vivo luminescence, IGSS, RBC promote NK cell activity and tumor cell hemagglutination assay and enzyme, etc. more than 10 kinds of new ways. The establishment of these methods, greatly enriched the means of cellular immunity detection methods, but also greatly promoted the development of basic theory and clinical studies of cellular immunity.

For autoimmune diseases, more research at home and abroad is systemic lupus erythematosus (SLE). Immuno-compromised patients, circulating immune complexes accumulate in the body, causing inflammation of tissue precipitation. RBC-C3b receptor rosette of low activity in SLE might be impaired C3b receptor, the receptor immune complexes covering vacancies and inhibitory factor receptor activity caused.

Disease of the blood system believes that blood is the main distributions of various immune cells, blood diseases occur inevitably affect the body's immune function. Studies have shown that blood cells in patients with immune function, C3b receptor rosette rate was significantly lower than that of ordinary people.

Immune cells can adhere to tumor cells, NK cells can enhance the ability to kill tumor cells, LAK cells, plays an important role in the anti-tumor immune responses. The patient's tumor disease can be reduced or CR1 function decreased, on the one hand to adjust cell tumor cells, and promote 
phagocytosis lower, on the other hand cause circulating immune complexes (CIC) is clear obstacles. NK cells to tumor cells lethal, cells are able to enhance this destruction, but when the animal suffering from cancer disease, which promote cell will be reduced.

In cardiovascular disease, the immune cell function in patients affected, not only serum C3 levels decline, and the body's ability to clear CIC also decreased cellular immune function disorder. Additionally, in clinical studies of endocrine, digestive, relationship to various diseases respiratory, nervous, urinary and other diseases, skin diseases, rheumatism, ENT diseases, pediatric diseases, infectious diseases and cellular immunity. Studies have shown that immune cell function in patients with varying degrees of decline.

Exercise can cause the body's immune function changes the fact that the academic community has been recognized. Now studies on exercise and cellular immune system relations, especially relations with the cellular immune adhesion function is increasing. After we have proved that humans and animals during the acute aerobic and anaerobic exercise, cell C3b receptor activity declined significantly lower their immune adhesion function. And long-term Tai Chi and qigong exercises can significantly enhance cell receptor activity, patients at different stages of tai chi and qigong exercises, cell receptor rosette rate has improved markedly, and finally stabilized at a certain level.

He has conducted research in all aspects of the blood and immune cells, spleen and cellular immunity, kidney and cellular immunity, and cellular immunity medicine, acupuncture and cellular immunity, blood viscosity and cell immunity. Cell generation, development and function is closely related to the spleen and kidney. Chinese medicine in cancer, autoimmune diseases (rheumatoid arthritis, nephrotic syndrome, I type diabetes, psoriasis) AIDS and emphysema treatment of acute infection and other diseases, the use of rosette assay results show significant RBC-C3bRR improve, significantly lower RBC-ICRR.

In the field of veterinary medicine, the immune cell research started late, but there are few data accumulation. Studies have shown that the prevalence of animals (such as infectious bursal disease, fowl cholera, Marek's disease, reticuloendotheliosis disease, coccidiosis) after, C3b receptors significantly reduced the number of cells, and immune complexes garland binding rate, different diseases have different manifestations, the serum also promote the presence of cellular immunity and inhibition factor, these two factor activity with age changes.

\section{Conclusion}

In summary, the proposed cellular immune function and the cellular immune system has already made great progress, but there are still many problems immune cells that we need to explore. Study on cellular immune function in both in basic research or in clinical application, we have important theoretical and practical value. I believe that in the near future, with the development of science and technology, cellular immunology, as a new discipline, will play an important role in the field of social production, the treatment of immune-depth study of the development of immune cells in the future will also be bring a revolution.

\section{REFERENCE:}

[1] Guo Feng, Yao Guangjin, Red Cell Immune Serum Assay Meng Xiangying Promoting Factors [J]. Shanghai Journal of Immunology, 1988,8 (6): 440.

[2] Nelson R A. The Immune Phenomenon: An Immunologically Specific Reaction between Microorganisms and Erythrocyte Leading to Enhanced Phagocytosis [J]. Science, 1953, 118, 773. 
[3] Nishioka K. Measurement of Complement by Agglutination of Human Erythrocyte Reacting in Immune-Adherence[J]. J Immunol,1963,90,86.

[4] Siegel I,Liu T, Gieicheer N. The Red-Cell Immune System [J]. Lancet, 1981, 8246(2): 556. 